\title{
Contour with Connected Components and Mesh Surface for Skin Image Segmentation
}

\author{
Hind Rostom Mohamed \\ Department of Computer, Mathematical and \\ Computer Sciences College, Al-Kufa University. \\ Al-Najaf City, Iraq.
}

\begin{abstract}
In the present paper, a Skin image segmentation method based on Contour with Connected Components and Mesh Surface is proposed.

The proposed scheme is tested successfully on a large set of images. The performance of the proposed detector compares favorably both computationally and qualitatively, in comparison contour detector with Connected Components and Mesh Surface which are also based on surround influence. The last stage includes Extraction of connected components skin image edge segmentation. The proposed scheme serves as a low cost preprocessing step for high level tasks, such as shape based recognition and image retrieval. The experimental results confirm the effectiveness of the proposed algorithm.
\end{abstract}

\section{General Terms}

Connected Components, Mesh Surface, Skin image segmentation

\section{Keywords}

Contour with Connected Components, Mesh Surface, Skin image, segmentation

\section{INTRODUCTION}

Human skin is the turbid media with multi-layered structure various pigments such as melanin and hemoglobin are contained in the media. The slight changes of the structure and pigment construction produce rich skin color variation [1]. Therefore, it is necessary to analyze the skin color based on the structure and pigment construction in reproducing and diagnosing the various skin colors. Skin segmentation in images is a theme that is present in many applications. This is the first step for image recognition.

Other application is for naked segmentation, in the internet [1].

Edge segmentation of an image reduces significantly the amount of data and filters out information that may be regarded as less relevant, preserving the important structural properties of an image. Thus, edges detected from its original image contain major information, which only needs a small amount of memory to store [7][9][16]. The purpose of detecting sharp changes in image brightness is to capture important events and changes in properties of the world [2].

Skin-color based face segmentation approaches have several advantages compared to other methods since under constant lighting conditions color is almost invariant against changes in

\author{
Ameer A. Mohammed Baqer Al-Shammaa \\ Center of Information Technology Research and \\ Development, Al-Kufa University. \\ Al-Najaf City, Iraq.
}

size, orientation and partial occlusion of the face. Moreover the processing of color information has proven to be much faster than processing other facial features which are an important point when dealing with video sequences [3]

Once the pixels of interest are located, unsupervised segmentation is used to separate these pixels into smaller regions which are homogeneous in color. This is a very important step because the skin segmentation will produce non-homogeneous regions. Often containing more than one objects [15]. Finally, a region merging step is introduced since the unsupervised segmentation can split the face regions into smaller homogeneous regions which have been greatly improved with respect to the work presented in [2][6][8][11].

The paper is organized as follows; Section 1 is an overview of the paper. Section 2 deals with the materials and methods, Section 3 illustrates the proposed approach and it results, while section 4 is dedicated to the conclusions arrived at.

\section{MATERIALS AND METHODS}

\subsection{Contour with Connected Components.}

Segmentation is about subdividing an image into its constituent regions or objects. The level up to which the subdivision is carried out depends on the problem being solved. A human skin color model is used to decide either a pixel is skin color or non-skin-color [5].

Image editing tasks normally involve one or more extended contours, not single edge elements in isolation. For this reason, contour-based image editing depends upon an efficient method for specifying a group of edges to which an action is to be applied.

An efficient method for grouping edges into closed contours has recently been reported. The algorithm consists of three main stages:

1. Line segment approximation.

2. Computation of posterior line grouping probabilities.

3. Shortest path computation of maximum-likelihood line segment cycles [17].

The next step is to remove small objects and to connect the connected components for skin image that has fewer than $P$ pixels, producing another binary image. The default connectivity is 8 for two dimensions. The statement is used $\mathrm{BW} 2=$ bwareaopen $(\mathrm{BW}, \mathrm{P}$, conn $)$ to specifies the desired 
connectivity . Where variable conn can have Value for Twodimensional connectivity:

\section{A) 4 if 4-connected neighborhood \\ B) 8 if 8-connected neighborhood}

The 1-valued elements define neighborhood locations relative to the central element of conn. Note that conn must be symmetric about its central element.

The basic steps for the desired connectivity are:

1- Determine the connected components: $\mathrm{CC}=$ bwconncomp $(\mathrm{BW}$, conn $)$;

2- Compute the area of each component: $\mathrm{S}=$ regionprops(CC, 'Area');

3- Remove small objects: $\mathrm{L}=$ labelmatrix $(\mathrm{CC})$

$$
\text { BW2 = ismember }(\mathrm{L} \text {, find }([\mathrm{S} \text {.Area }]>=\mathrm{P})) \text {; }
$$

The next step find connected components in binary Skin image .The basic steps in finding the connected components are:

1- Searching for the next unlabeled pixel, $\mathrm{p}$.

2- Using a flood-fill algorithm to label all the pixels in the connected component containing $\mathrm{p}$.

3- Repeating steps 1 and 2 until all the pixels are labeled.

The components embrace four structure fields:

1- Connectivity: Connectivity of the connected components (objects).

2- ImageSize: Size of image.

3- NumObjects: Number of connected components (objects) in image.

4- PixelIdxList: 1-by-NumObjects cell array where the kth element in the cell array is a vector containing the linear indices of the pixels in the kth object.

\subsection{Mesh Surface segmentation}

Any set of points in the $\mathrm{x}, \mathrm{y}$-plane can be triangulated by the Delaunay algorithm. In the physical model, the edges of the triangles (the connections between pairs of points) correspond to bars, and the points correspond to joints of the truss[13]. Each bar has a force-displacement relationship $f\left(l, l_{0}\right)$ depending on its current length $l$ and its unextended length $l_{0}$. The external forces on the structure come at the boundaries. At every boundary node, there is a reaction force acting normal to the boundary. The magnitude of this force is just large enough to keep the node from moving outside. The positions of the joints (these positions are our principal unknowns) are found by solving for a static force equilibrium in the structure. The hope is that (when $\mathrm{h}(\mathrm{x}, \mathrm{y})=1$ ) the lengths of all the bars at equilibrium will be nearly equal, giving a well-shaped triangular mesh [18]. To solve for the force equilibrium, collect the $\mathrm{x}$ - and $\mathrm{y}$-coordinates of all $\mathrm{N}$ mesh points into an N-by-2 array p:

$$
P=\left[\begin{array}{ll}
x & y
\end{array}\right]
$$

The force vector $\mathrm{F}(\mathrm{p})$ has horizontal and vertical components at each mesh point:

$$
F(p)=\left[F_{\text {int }, x}(p) \quad F_{\text {int }, y}(p)\right]+\left[F_{\text {ext }, x}(p) \quad F_{\text {ext }, y}(p)\right]
$$

Where $\mathrm{F}_{\text {int }}$ contains the internal forces from the bars, and $\mathrm{F}_{\text {ext }}$ are the external forces reactions from the boundaries. The first column of $\mathrm{F}$ contains the $\mathrm{x}$-components of the forces, and the second column contains the y-components.

Now the description to steps 1 to 8 in the distmesh2d algorithm [19]:

1. The first step creates a uniform distribution of nodes within the bounding box of the geometry, corresponding to equilateral triangles.

2. The next step removes all nodes outside the desired geometry.

3. Now, the code enters the main loop, where the location of the $\mathrm{N}$ points is iteratively improved. the variable pold is initialized for the first iteration, and start the loop.

4. The list of triangles $t$ is an array with 3 columns. Each row represents a triangle by three integer. In creating a list of edges, each triangle contributes three node pairs. Since most pairs will appear twice (the edges are in two triangles), duplicates have to be removed.

5. The next two lines give graphical output after each re-triangulation.

6. Each bar is a two-component and its length is in L

7. If a point ends up outside the geometry after the update of p. It is moved back to the closest point on the boundary (using the distance function). This corresponds to a reaction force normal to the boundary. Points are allowed to move tangentially along the boundary.

8. Finally, the termination criterion is based on the maximum node movement in the current iteration (excluding the boundary points).

The elastic matching of minutia is achieved by placing a bounding box around each template minutia.

\section{DESIGN APPROACH AND RESULTS}

Image segmentation is the process of partitioning /subdividing a digital image into multiple meaningful regions or sets of pixels regions with respect to a particular application [1]. The segmentation is based on measurements taken from the image and might be grey level, colour, texture, depth or motion. The result of image segmentation is a set of segments that collectively cover the entire image. All the pixels in region are similar with respect to some characteristic or computed property, such as colour, intensity, or texture. Adjacent regions differ with respect to same characteristics. Edge segmentation is one of the frequently used techniques in digital image processing [10]. In this research used two color image databases:

(1) Database prepared in our conditions, images obtained from in Al-Sder Hospital.

(2) Skin database [4] and some other images obtained from internet. 
(see Figure 1) shown skin library samples, (A) Samples with Skin diseases (B) Samples with other diseases.
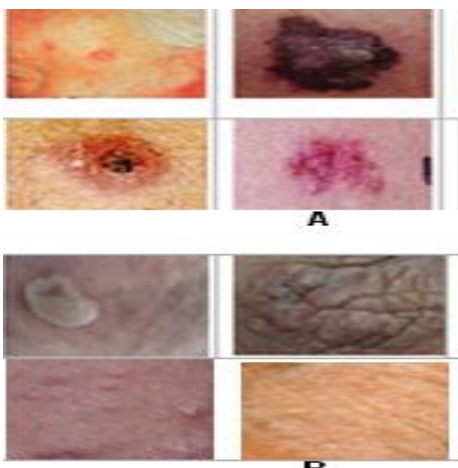
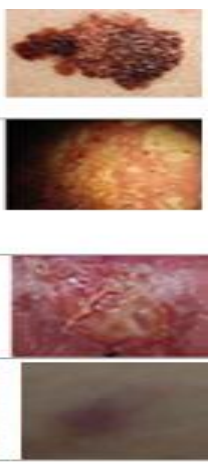

Fig 1: The skin library samples, (A) Samples with Skin diseases (B) Samples with other diseases.

In this section a detailed experimental comparison of the above stated (see Figure 2) shows Contour Images.

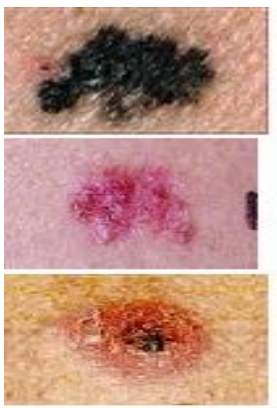

A

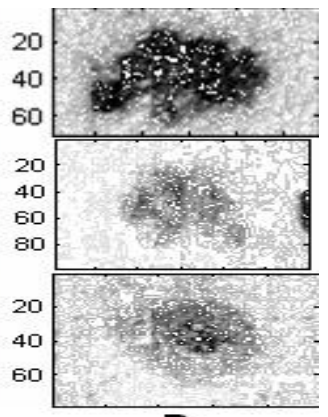

B
Fig 2: (A) Original Skin Image, (B) Contour Image.

(see Figure 3) shows Center points for some types of skin image.

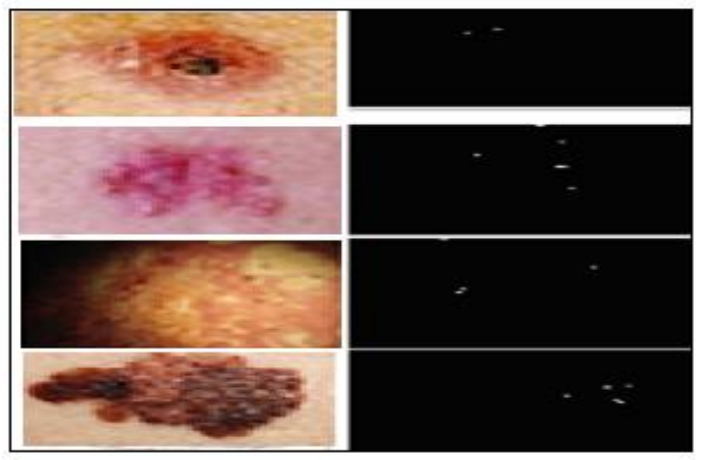

Fig 3: Center points for some types of skin image.

(see Figure 4) shows Edge for all region and Edge for Center Skin Image.
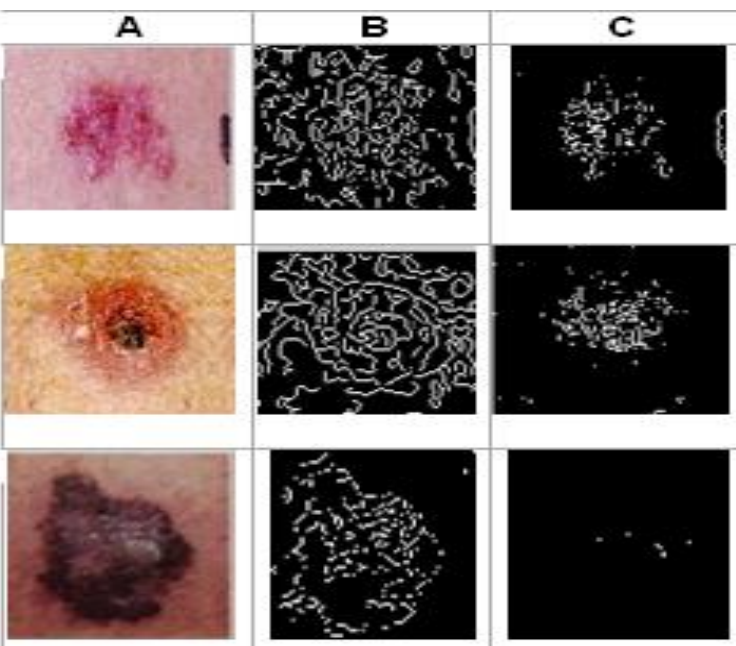

Fig 4: (A) Original Skin Image, (B) Edge for all regions, (C) Edge for Center Skin Image.

(see Figure 5) shows Connected Components for center region for each Skin image type.

After extracting boundary of images regions have to be identified for medical purposes. then to focus on particular area like tumors regions has to be filled to highlight that particular area the results, are minimally connected lines that form equidistant boundaries between the objects[12][13]. (see Figure 6) shown Mesh Surface skin image segmentation.

The objective in this stage is to reject regions which have no holes as mentioned in the above region.

To avoid the complexity and cost of the non-linear optimization process described above, First, resample each irregular polygonal patch into a regular grid of points (the spring mesh)[14]. Then apply girded data fitting techniques to this spring mesh to obtain a spline approximation. The advantage of these techniques is that they avoid the parameter re-estimation step described earlier and are hence significantly faster. It is worth pointing out that in the applied application there is nothing sacrosanct about the original mesh vertices [20]. In particular, the vertices produced by the applied range image integration method are at a scale that approaches the noise-limited resolution of our sensor.

The only constraints are that it must be rectangularly parameterizable and must not have holes. These are reasonable assumptions since the models input to the proposed system are seamless or can easily be made so by acquiring and integrating more scans and by recent holefilling techniques [21]. The research goal is to generate a uniform grid of points over the polygonal surface that samples the surface well shown in (see Figure 7) with skin image boundaries components edge segmentation. 


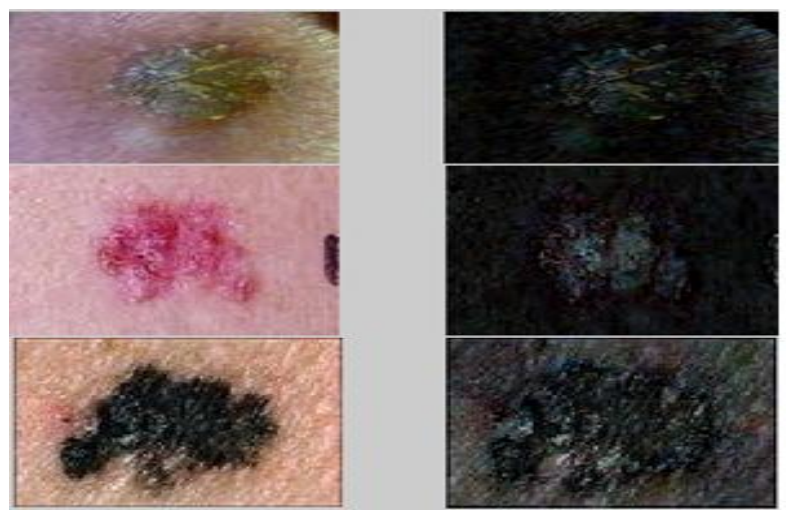

Fig 5: Connected Components for center region for each Skin image type
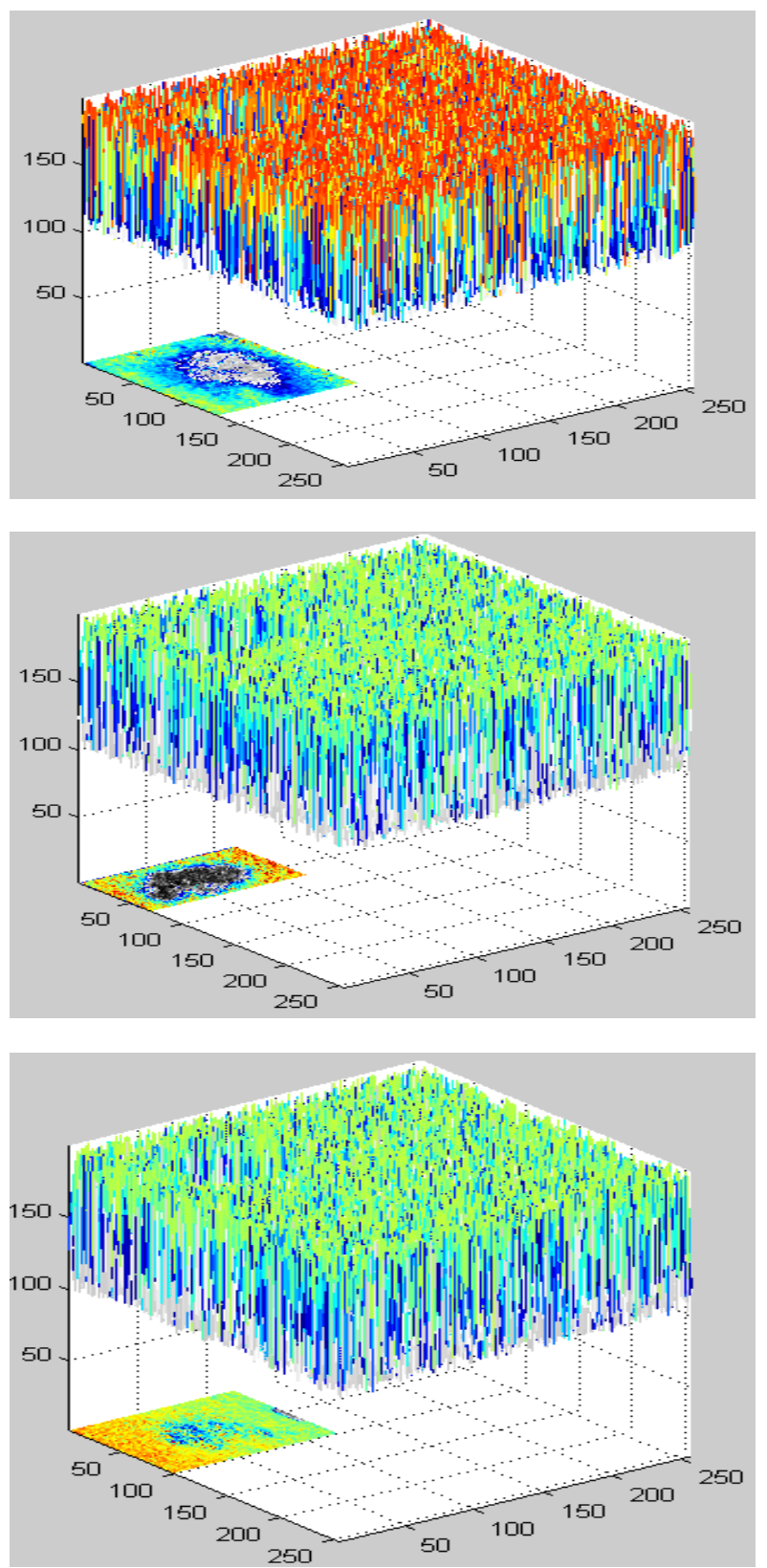

Fig 6: Mesh Surface skin image segmentation.
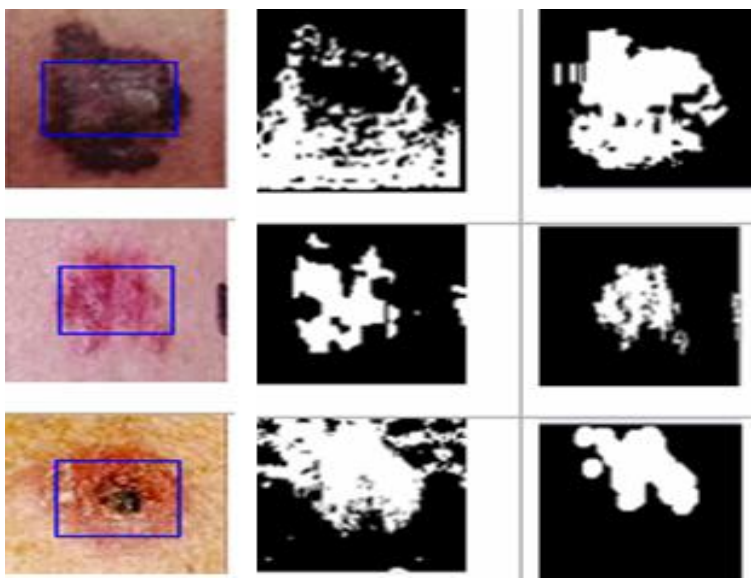

Fig 7: skin image boundaries components edge segmentation

\section{CONCLUSION}

The research work proposed a new goodness criterion for segmenting closed figures. The propose approach for Mean Shift Algorithm and contour Image based segmentation has been demonstrated, but it should be straightforward to extend it to incorporate other features.

The proposed approach is a region based one and applies most naturally to closed contours. It can be extended to open contours as long as the contour can be considered as organizing the image into distinct regions. Components have been connected for mean shift results by removing small objects and connecting the connected components for skin image that has fewer than $\mathrm{P}$ pixels, producing another binary image .

Finally, the object tracking process performs as memory for collecting skin-color objects obtained from previous frame to guide the next frame in order to remove skin-color pixels that immediately appear frame by frame. The experimental results show reliable satisfying subjective test results. It can be concluded that the present algorithm demonstrate the super performance with respect to speed, zero repeats, low false positive rate and high accuracy.

\section{REFERENCES}

[1] Norimichi Tsumura, Hideaki Haneishi and Yoichi Miyake, 1999. Independent Component Analysis of Skin Color Image. The Sixth Color Imaging Conference: Color Science, Systems, and Applications, Vol. 16, Issue 9, pp. 2169-2176.

[2] C. NagaRaju , S. NagaMani, G. rakesh Prasad, S. Sunitha. 2011. Morphological Edge Detection Algorithm Based on Multi-Structure Elements of Different Directions, International Journal of Information and Communication Technology Research, Volume 1, No. 1, P.P. 37- 43, May 2011.

[3] Luis Torres , Edward J. Delp, 2001. An unsupervised color image segmentation algorithm for face segmentation applications, Image Processing, IEEE International Conference - ICIP , P.P. 681-684. 
[4] Phung, S. L., Bouzerdoum, A., And Chai, D. 2002. A novel skin color model in ycbcr color space and its application to human face segmentation. In IEEE International Conference on Image Processing (ICIP' 2002), vol. 1, P.P. 289-292.

[5] Teerayoot Sawangsri, Vorapoj Patanavijit, and Somchai Jitapunkul. 2007. Face Segmentation Using Novel SkinColor Map And Morphological Technique. World Academy of Science, vol.2 (95), P.P. 534-537.

[6] Quan Huynh-Thu Mitsuhiko Meguro Masahide Kaneko. 2002. Skin-Color-Based Image Segmentation and Its Application in Face Segmentation, IAPR Workshop on Machine Vision Applications, Proceedings of MVA 2002 IAPR Workshop, Machine vision applications, P.P. 48-51.

[7] Y. Ramadevi. 2011. A novel threshold based edge Detection algorithm. International Journal of Engineering Science and Technology (IJEST), Vol. 3 No. 6, P.P. $4534-4538$.

[8] Jianguo Wang, Tieniu Tan. 2000. A new face segmentation method based on shape information. Journal Pattern Recognition Letters- Elsevier Science Inc. New York, NY, USA, Vol. 21 Issue 6-7, P.P. 463 471.

[9] M. Elad et al. 2002. Rejection based classifier for face Detection. Pattern Recognition Letters. vol. 23, P.P. 1459-1471.

[10] B.Poornima, Y.Ramadevi, T.Sridevi. 2011. Threshold Based Edge Detection Algorithm. ACSIT International Journal of Engineering and Technology, Vol.3, No.4, August 2011.

[11] M Rama Bai 1, V Venkata Krishna J SreeDevi. 2010. New Morphological Approach for Noise Removal cum Edge Detection. IJCSI International Journal of Computer Science Issues, Vol. 7, Issue 6, November 2010.

[12] K. V. Joshi, G H Patel, 2011. Edge Detection and Template ,Matching Approaches for Human Ear
Segmentation. International Conference on Intelligent Systems and Data Processing (ICISD), Special Issue published by International Journal of Computer Applications (IJCA), P.P. 50-55.

[13] Ming-Hsuan Yang et.al. 2002. Detecting faces in Images: a survey. IEEE transaction on Pattern analysis and machine intelligene, vol. 24, no.1.

[14] Sanjay singh et.al, 2003. A robust skin color based face segmentation algorithm. Tamkang Journal of Science and Engineering, vol.6, no.4, P.P. 227-234.

[15] Dr. Arti Khaparde*, Sowmya Reddy.Y Swetha Ravipudi. 2003. Face Detection Using Color Based Segmentation and Morphological Processing : A Case Study. Bharath Institute of Science and Technology.

[16] Michael Padilla and Zihong Fan, 2003. EE368 Digital Image Processing Project Automatic Face Segmentation Using Color Based Segmentation and Template/Energy Thresholding, EE368 - Dr. B. Girod, Stanford University, Spring, P.P. 1-7.

[17] James H. Elder, Member, IEEE, And Richard M. Goldberg, 2001. Image Editing In The Contour Domain. IEEE TRANSACTIONS ON PATTERN ANALYSIS AND MACHINE INTELLIGENCE, Vol. 23, No. 3, P.P. 291-296.

[18] S.-W. Cheng, T. K. Dey, H. Edelsbrunner, M. A. Facello, and S.-H. Teng, 1999. Sliver exudation, in Symposium on Computational Geometry, P.P. 1-13.

[19] Per-Olof Persson And Gilbert Strang, 2005. A Simple Mesh Generator In Matlab. Per-Olof Persson And Gilbert Strang.

[20] David R.Forsey and Richard H. Bartels. 1991. Surface fitting with hierarchical splines. In Topics in the Construction, Manipulation, and Assessment of Spline Surfaces, SIGGRAPH course 25, pages 7-0-7-14.

[21] Gerald Farin. 1990. Curves and Surfaces for Computer Aided Geometric Design. Academic Press. 